\title{
Embeddings of finite-dimensional operator spaces into the second dual
}

\author{
by \\ Alvaro Arias (Denver, CO) and Timur Oikhberg (Irvine, CA)
}

\begin{abstract}
We show that, if a a finite-dimensional operator space $E$ is such that $X$ contains $E C$-completely isomorphically whenever $X^{* *}$ contains $E$ completely isometrically, then $E$ is $2^{15} C^{11}$-completely isomorphic to $\mathbf{R}_{m} \oplus \mathbf{C}_{n}$ for some $n, m \in \mathbb{N} \cup\{0\}$. The converse is also true: if $X^{* *}$ contains $\mathbf{R}_{m} \oplus \mathbf{C}_{n} \lambda$-completely isomorphically, then $X$ contains $\mathbf{R}_{m} \oplus \mathbf{C}_{n}(2 \lambda+\varepsilon)$-completely isomorphically for any $\varepsilon>0$.
\end{abstract}

1. Introduction. Local reflexivity of Banach spaces was first discovered by J. Lindenstrauss and H. Rosenthal in [12]. Later, W. Johnson, H. Rosenthal, and M. Zippin [9] improved on this result, and obtained:

Theorem 1.1. Suppose $X$ is a Banach space, $E$ and $F$ are finite-dimensional subspaces of $X^{* *}$ and $X^{*}$, respectively, and $\varepsilon>0$. Then there exists an operator $u: E \rightarrow X$ such that $\|u\|<1+\varepsilon,\left.u\right|_{E \cap X}=I_{E \cap X}$, and $\langle u e, f\rangle=$ $\langle e, f\rangle$ for any $e \in E$ and $f \in F$.

This immediately implies the result of [12]:

Corollary 1.2. Suppose $E$ and $X$ are Banach spaces, $E$ is a finitedimensional space, and $E$ is contained in $X^{* *} C$-isomorphically (that is, there exists $E^{\prime} \hookrightarrow X^{* *}$ such that $\left.d\left(E, E^{\prime}\right) \leq C\right)$. Then $E$ is contained in $X$ $(C+\varepsilon)$-isomorphically for any $\varepsilon>0$.

In the non-commutative case, the results quoted above do not hold in general. It is well known that an infinite-dimensional operator space need not be locally reflexive. Moreover, for every $n>2$ the space $\ell_{1}^{n}$ (equipped with the maximal operator space structure) is contained in $\mathbf{B}=\mathbf{K}^{* *}$, while, by Theorem 21.5 of $[16], d_{\mathrm{cb}}\left(\ell_{1}^{n}, E\right) \geq n /(2 \sqrt{n-1})$ for any $E \hookrightarrow \mathbf{K}$ (here and below, $\mathbf{B}$ and $\mathbf{K}$ denote the spaces of bounded and compact operators on $\ell_{2}$, respectively).

2000 Mathematics Subject Classification: 46L07, 47L25.

Key words and phrases: local reflexivity, duality of operator spaces.

Second author supported in part by the NSF grant DMS-0500957. 
In this paper, we show that a "non-commutative" analogue of Corollary 1.2 holds for a finite-dimensional operator space $E$ if and only if $E$ is completely isomorphic to a direct sum of row and column spaces (Theorem 1.3). We say that $X$ contains $E C$-completely isomorphically $(C$-ci) if there exists $F \hookrightarrow X$ such that $d_{\mathrm{cb}}(E, F) \leq C$; and $X$ is said to contain $E$ $C+$-ci if it contains $E C_{1}$-ci for every $C_{1}>C$. A finite-dimensional operator space $E$ is said to be $C$-bidually representable $(C$-BDR, for short) if an operator space $X$ contains $E C$-completely isomorphically whenever $X^{* *}$ contains a completely isometric copy of $E$; and $E$ is said to be $C+-B D R$ if it is $C_{1}$-BDR for any $C_{1}>C$.

Below, $\mathbf{R}_{n}$ and $\mathbf{C}_{n}$ stand for the spaces spanned by the first row and the first column of $n \times n$ matrices, respectively. $\oplus$ means $\oplus_{\infty}$ (the $\ell_{\infty}$ sum of spaces), unless specified otherwise.

THEOREM 1.3.

(1) If $E$ is $\lambda$-completely isomorphic to $\mathbf{R}_{m} \oplus \mathbf{C}_{n}$ for some $m, n \in \mathbb{Z}_{+}$, then $E$ is $2 \lambda^{2}+-B D R$.

(2) If $E$ is $\lambda+-B D R$, then, for some $m, n \in \mathbb{Z}_{+}, d_{\mathrm{cb}}\left(E, \mathbf{R}_{m} \oplus \mathbf{C}_{n}\right) \leq$ $2^{15} \lambda^{11}$

THEOREM 1.4. An operator space is 1-BDR if and only if it is 1-dimensional.

The rest of the paper is organized as follows: in Section 2, we gather some essential facts about non-dual local reflexivity, and prove item (1) of Theorem 1.3. The proof of Theorem 1.3(2) proceeds in two steps. First, we show that $E$ embeds "nicely" into $\mathbf{R} \oplus \mathbf{C}$ (Section 3 ). In Section 4 we complete the proof of Theorem 1.3(2). Section 5 is devoted to proving Theorem 1.4. Finally, in Section 6, we consider our problem in the setting of $C^{*}$-algebras.

The notation used in this paper is, by and large, either standard, or explained above. The minimal (also called injective, or spatial) tensor product of operator spaces is denoted by $\otimes$. If $T: X \rightarrow Y$ is a finite rank operator, $\widetilde{T}$ stands for the corresponding element of $X^{*} \otimes Y$ (then $\left.\|T\|_{\mathrm{cb}}=\|\widetilde{T}\|\right)$. We often use $\mathbf{M}_{n}, \mathbf{B}, \mathbf{K}$, and $\mathbf{K}_{0}$ - the spaces of $n \times n$ matrices, of bounded operators on $\ell_{2}$, of compact operators on $\ell_{2}$, and of compact operators with matrices having finitely many non-zero entries, respectively.

In the proofs, we use the notion of exactness of an operator space, and the notion of a complete $M$-ideal in an operator space.

We say that an operator space $Z$ is $C$-exact $(C>0)$ if, for any finitedimensional subspace $E \hookrightarrow Z$, and every $\varepsilon>0$, there exist $N \in \mathbb{N}$ and $F \hookrightarrow M_{N}$ with $d_{\mathrm{cb}}(E, F)<C+\varepsilon$; and $Z$ is said to be exact if it is $C$-exact for some $C$. The exactness constant of $Z$ (denoted by $\operatorname{ex}(Z)$ ) is the infimum of all the $C$ 's with the above property. It is easy to see that the row space $\mathbf{R}$, 
the column space $\mathbf{C}$, and the space of compact operators $\mathbf{K}$ are 1-exact. On the other hand, it is known that $\mathbf{B}$ is not exact. The reader is referred to [15], Chapter 17 of [16], or Chapter 14 of [6] for more information.

A subspace $X$ of an operator space $Y$ is called a complete $M$-summand if $Y=Y \oplus_{\infty} Z$ for some $Z \hookrightarrow Y$; and $X$ is a complete $M$-ideal in $Y$ if $X^{\perp \perp}$ is a complete $M$-summand in $Y^{* *}$. We refer the reader to [5], or to Section 4.8 of [2], for information about complete $M$-ideals. For the theory of $M$-ideals in Banach spaces, see [8].

Finally, $\mathbb{Z}_{+}=\mathbb{N} \cup\{0\}$ is the set of non-negative integers.

2. Some remarks on bidual representability. To prove Theorem $1.3(1)$, we begin with

Proposition 2.1. Suppose $E$ and $F$ are finite-dimensional operator spaces, $X$ is an operator space, $u: E \rightarrow X^{* *}$ and $v: X^{* *} \rightarrow F$ are linear maps, and $\varepsilon>0$. Then there exist linear maps $u_{1}: E \rightarrow X$ and $v_{1}: X \rightarrow F$ such that $\left\|u_{1}\right\|_{\mathrm{cb}}\left\|v_{1}\right\|_{\mathrm{cb}}<(1+\varepsilon) \operatorname{ex}\left(E^{*}\right) \operatorname{ex}(F)\|u\|_{\mathrm{cb}}\|v\|_{\mathrm{cb}}$ and $v_{1} u_{1}=v u$.

Proof. Pick biorthogonal systems $\left(e_{i}, e_{i}^{*}\right)_{i=1}^{n}$ and $\left(f_{j}, f_{j}^{*}\right)_{j=1}^{m}$ in $E$ and $F$, respectively. Write $\widetilde{u}=\sum_{i} e_{i}^{*} \otimes x_{i}^{* *}$ and $\widetilde{v}=\sum_{j} f_{j} \otimes x_{j}^{* * *}$ with $x_{i}^{* *} \in X^{* *}$ and $x_{j}^{* * *} \in X^{* * *}$. By Proposition 3.2.1 of [6], $\left(\mathbf{M}_{N}(X)\right)^{* *}=\mathbf{M}_{N}\left(X^{* *}\right)$ isometrically, hence $\widetilde{v} \in X^{* * *} \otimes F$ can be "approximated" by $\widetilde{v}_{1}=\sum_{j} f_{j} \otimes x_{j}^{*} \in$ $F \otimes X^{*}$ in such a way that $(1)\left\|\widetilde{v}_{1}\right\|<\sqrt{1+\varepsilon} \operatorname{ex}(F)\|\widetilde{v}\|$, and $(2)\left\langle x_{j}^{*}, x_{i}^{* *}\right\rangle=$ $\left\langle x_{j}^{* * *}, x_{i}^{* *}\right\rangle$ for any pair $(i, j)$. Similarly, there exists $\widetilde{u}_{1}=\sum_{i} e_{i}^{*} \otimes x_{i} \in E^{*} \otimes X$ such that $\left\|\widetilde{u}_{1}\right\| \leq \sqrt{1+\varepsilon} \operatorname{ex}\left(E^{*}\right)\|\widetilde{u}\|$, and $\left\langle x_{j}^{*}, x_{i}^{* *}\right\rangle=\left\langle x_{j}^{*}, x_{i}\right\rangle$ for any pair $(i, j)$.

Now go back from tensor products to c.b. maps $u_{1}: E \rightarrow X$ and $v_{1}: X \rightarrow F$. By the above, $\left\|u_{1}\right\|_{\mathrm{cb}}<\sqrt{1+\varepsilon} \operatorname{ex}\left(E^{*}\right)\|u\|_{\mathrm{cb}}$ and $\left\|v_{1}\right\|_{\mathrm{cb}}<$ $\sqrt{1+\varepsilon} \operatorname{ex}(F)\|v\|_{\mathrm{cb}}$. Moreover, for any $i$,

$$
v_{1} u_{1} e_{i}=\sum_{j}\left\langle x_{j}^{*}, x_{i}\right\rangle f_{j}=\sum_{j}\left\langle x_{j}^{* * *}, x_{i}^{* *}\right\rangle f_{j}=v u e_{i} .
$$

Therefore, $v u=v_{1} u_{1}$ and $\left\|u_{1}\right\|_{\mathrm{cb}}\left\|v_{1}\right\|_{\mathrm{cb}}<(1+\varepsilon) \operatorname{ex}\left(E^{*}\right) \operatorname{ex}(F)\|u\|_{\mathrm{cb}}\|v\|_{\mathrm{cb}}$.

Proposition 2.1 implies:

Corollary 2.2. Suppose $X$ is an operator space, and $\delta>0$. Then:

(1) If $X^{* *}$ contains a $\lambda$-injective finite-dimensional operator space $E C$ completely isomorphically, then $X$ contains $E\left(\lambda C \operatorname{ex}(E) \operatorname{ex}\left(E^{*}\right)+\delta\right)$ completely isomorphically.

(2) If $X^{* *}$ contains $\mathbf{R}_{m} \oplus \mathbf{C}_{n}\left(n, m \in \mathbb{Z}_{+}\right)$C-completely isomorphically, then $X$ contains $\mathbf{R}_{m} \oplus \mathbf{C}_{n}(2 C+\delta)$-completely isomorphically. Consequently, $\mathbf{R}_{m} \oplus \mathbf{C}_{n}$ is $2+-B D R$. 
(3) Suppose $E=\mathbf{R}_{m}, \mathbf{C}_{m}, \ell_{\infty}^{2}, \mathbb{C} \oplus_{\infty} \mathbf{R}_{m}$, or $\mathbb{C} \oplus_{\infty} \mathbf{R}_{m}(m \in \mathbb{N})$. If $X^{* *}$ contains $E C$-completely isomorphically, then $X$ contains $E$ $(C+\delta)$-completely isomorphically. Consequently, $E$ is $1+-B D R$.

Proof. (1) Consider a subspace $E^{\prime} \hookrightarrow X^{* *}$ for which there exists a completely contractive isomorphism $u E \rightarrow E^{\prime}$ with $\left\|u^{-1}\right\|_{\mathrm{cb}} \leq C$. As $E$ is $\lambda$ injective, there exists $v: X^{* *} \rightarrow E$ extending $u^{-1}$ (that is, $\left.v\right|_{E^{\prime}}=u^{-1}$ ) of norm not exceeding $\lambda C$. By Proposition 2.1, for every $\varepsilon>0$ there exist $u_{1}$ : $E \rightarrow X$ and $v_{1}: X \rightarrow E$ such that $\left\|u_{1}\right\|_{\mathrm{cb}}\left\|v_{1}\right\|_{\mathrm{cb}} \leq(1+\varepsilon) \operatorname{ex}(E) \operatorname{ex}\left(E^{*}\right) \lambda C$ and $v_{1} u_{1}=v u=I_{E}$. Therefore, $d_{\mathrm{cb}}\left(E, u_{1}(E)\right) \leq(1+\varepsilon) \operatorname{ex}(E) \operatorname{ex}\left(E^{*}\right) \lambda C$. Since $\varepsilon$ can be arbitrarily small, we are done.

(2) The space $\mathbf{R}_{m} \oplus \mathbf{C}_{n}$ embeds into $\mathbf{M}_{m+n}$ as a 1-completely complemented subspace, hence $\operatorname{ex}\left(\mathbf{R}_{m} \oplus \mathbf{C}_{n}\right)=1$. Moreover, $\left(\mathbf{R}_{m} \oplus \mathbf{C}_{n}\right)^{*}=$ $\mathbf{C}_{n} \oplus_{1} \mathbf{R}_{m}$ is 2-completely isomorphic to $\mathbf{C}_{n} \oplus \mathbf{R}_{m}$, hence $\operatorname{ex}\left(\left(\mathbf{R}_{m} \oplus \mathbf{C}_{n}\right)^{*}\right) \leq 2$. An application of part (1) yields the desired result.

(3) Reason as in the proof of part (2), and recall that $\operatorname{ex}(E)=\operatorname{ex}\left(E^{*}\right)=1$ for any $E$ from the list (see Chapter 21 of [16]).

REMARK 2.3. We return to the connections between injectivity, exactness, and BDR in Section 5. Meanwhile, note that by Theorem 1.4, $\mathbb{C}$ is the only 1-BDR space. Hence, the condition of being 1-BDR is much stronger than being $1+-$ BDR.

The next result shows that bidual representability is an isomorphic property.

Proposition 2.4. Suppose $X$ is an operator space, $E$ is a finite-dimensional subspace of $X^{* *}, d_{\mathrm{cb}}(E, F)=C_{1}$, and $\operatorname{ex}(F)<C_{2}$. Then there exists an operator space $Y$ such that $d_{\mathrm{cb}}(X, Y)<C_{1} C_{2}$ and $Y^{* *}$ contains a completely isometric copy of $F$.

Proof. Suppose the map $u: E \rightarrow F$ is such that $\|u\|_{\mathrm{cb}}=C_{1}$ and $\left\|u^{-1}\right\|_{\mathrm{cb}}=1$. By renorming $X$, we shall construct a space $Y$ and a map $T: X \rightarrow Y$ such that $\|T\|_{\mathrm{cb}}<C_{1} C_{2}$, and $T^{-1}$ is completely contractive. To this end, find a subspace $F_{1} \hookrightarrow \mathbf{M}_{N}$ and a map $v: F_{1} \rightarrow F$ such that $\left\|v^{-1}\right\|_{\mathrm{cb}}=1$ and $\|v\|_{\mathrm{cb}}<C_{2}$. Embedding $F$ into $\mathbf{B}$ completely isometrically, and applying Stinespring's extension theorem, we obtain an operator $\widetilde{v}: \mathbf{M}_{N} \rightarrow \mathbf{B}$ such that $\left.\widetilde{v}\right|_{F_{1}}=v$ and $\|\widetilde{v}\|_{\mathrm{cb}}=\|v\|_{\mathrm{cb}}$. Let $\widetilde{F}=\widetilde{v}\left(\mathbf{M}_{N}\right)$.

Extend $w=v^{-1} u$ to $\widetilde{w}: X^{* *} \rightarrow \mathbf{M}_{N}$ such that $\|\widetilde{w}\|_{\mathrm{cb}}=\|w\|_{\mathrm{cb}} \leq C_{1}$ and $\left.\widetilde{w}\right|_{E}=w$. Let $C_{1}^{\prime}=C_{1} C_{2} /\|v\|_{\mathrm{cb}}$. The unit ball of $X^{*} \otimes \mathbf{M}_{N}$ is weak* dense in the unit ball of $X^{* * *} \otimes \mathbf{M}_{N}$, hence there exists an operator $w_{1}: X \rightarrow \mathbf{M}_{N}$ such that $\left\|w_{1}\right\|_{\mathrm{cb}}<C_{1}^{\prime}$ and $\left.w_{1}^{* *}\right|_{E}=w$.

Define a new operator space structure $Y$ on $X$ by setting, for $x \in X \otimes \mathbf{K}_{0}$,

$$
\|x\|_{Y \otimes \mathbf{K}}=\max \left\{\|x\|_{X \otimes \mathbf{K}},\left\|\left(\widetilde{v} w_{1} \otimes I_{\mathbf{K}}\right) x\right\|_{\widetilde{F} \otimes \mathbf{K}}\right\} .
$$


Denote by $T$ the formal identity map from $X$ to $Y$. Clearly, $T^{-1}$ is a complete contraction, and $\|T\|_{\mathrm{cb}} \leq\left\|\widetilde{v} w_{1}\right\|_{\mathrm{cb}}<C_{1} C_{2}$. Moreover, for $x^{* *} \in X^{* *} \otimes \mathbf{K}_{0}$,

$$
\left\|x^{* *}\right\|_{Y^{* *} \otimes \mathbf{K}}=\max \left\{\left\|x^{* *}\right\|_{X^{* *} \otimes \mathbf{K}},\left\|\left(\widetilde{v} w_{1}^{* *} \otimes I_{\mathbf{K}}\right) x^{* *}\right\|_{\widetilde{F} \otimes \mathbf{K}}\right\},
$$

which implies that, for $e \in E \otimes \mathbf{K}_{0},\|e\|_{Y^{* *} \otimes \mathbf{K}}=\left\|\left(u \otimes I_{\mathbf{K}}\right) e\right\|_{F \otimes \mathbf{K}}$.

Proposition 2.5. Suppose the space $E$ is $C+-B D R$. Then $E$ is $C$-exact.

Proof. Apply the definition of BDR to $X=\mathbf{K}$.

Corollary 2.6. Suppose $E$ and $E^{\prime}$ are operator spaces of the same (finite) dimension, $E$ is $C-B D R, d_{\mathrm{cb}}\left(E, E^{\prime}\right)=\lambda$, and $\operatorname{ex}(E)<\mu$. Then an operator space $X$ contains $E C \lambda \mu$-completely isomorphically whenever $X^{* *}$ contains $E^{\prime}$ completely isometrically. Consequently, $E^{\prime}$ is $C \lambda^{2} \mu-B D R$.

Proof. Suppose $E^{\prime}$ is contained in $X^{* *}$. Consider a map $u: E^{\prime} \rightarrow E$ such that $\|u\|_{\mathrm{cb}}=\lambda$ and $\left\|u^{-1}\right\|_{\mathrm{cb}}=1$. By the proof of Proposition 2.4, there exists an operator space $Y$ and a map $T: X \rightarrow Y$ such that $\|T\|_{\mathrm{cb}}<\lambda \mu$, $\left\|T^{-1}\right\|_{\mathrm{cb}} \leq 1$, and $T^{* *}\left(E^{\prime}\right)=E$. There exists a subspace $F \hookrightarrow Y$ with $d_{\mathrm{cb}}(E, F) \leq C$. Let $F^{\prime}=T^{-1}(F) \hookrightarrow X$. Then

$$
d_{\mathrm{cb}}\left(E, F^{\prime}\right) \leq d_{\mathrm{cb}}(E, F) d_{\mathrm{cb}}\left(F, F^{\prime}\right) \leq d_{\mathrm{cb}}(E, F)\|T\|_{\mathrm{cb}}\left\|T^{-1}\right\|_{\mathrm{cb}}<C \lambda \mu .
$$

Therefore, $d_{\mathrm{cb}}\left(E^{\prime}, F^{\prime}\right) \leq d_{\mathrm{cb}}\left(E^{\prime}, E\right) d_{\mathrm{cb}}\left(E, F^{\prime}\right)<C \lambda^{2} \mu$.

3. Proof of Theorem 1.3(2): $E$ is a subspace of $\mathbf{R} \oplus \mathbf{C}$. In this section, we make the first step toward proving Theorem 1.3(2) by showing that every $C$-BDR space embeds "neatly" into $\mathbf{R} \oplus \mathbf{C}$. More precisely, we prove:

Theorem 3.1. For every $N \in \mathbb{N}$, there exists a separable operator space $X$ such that:

(1) $X^{* *}$ contains $\mathbf{B}$ as a complete $M$-summand.

(2) Suppose $E$ is a finite-dimensional operator space such that $d_{\mathrm{cb}}\left(E, E^{\prime}\right)$ $<C$ for some $E^{\prime} \hookrightarrow \ell_{\infty}\left(\mathbf{M}_{N}\right)$, and $X$ contains $E$ c-completely isomorphically for some $c<C$. Then $E$ is $4 \sqrt{2} C^{3}$-completely isomorphic to a subspace of $\mathbf{R} \oplus \mathbf{C}$.

Consequently, any $C+-B D R$ space is $4 \sqrt{2} C^{3}$-completely isomorphic to a subspace of $\mathbf{R} \oplus \mathbf{C}$.

We start the proof by constructing the space $X$. At the Banach space level, let $X=\left(\bigoplus_{n>N} \mathbf{M}_{n}\right)_{c}$ be the space of all sequences whose elements are $n \times n$ matrices, and which have a limit (in $\mathbf{K}$ ). Denote by $P_{n}$ (once again, $n>N)$ the canonical "truncation" from $X$ to $\left(\sum_{k=N+1}^{n} \mathbf{M}_{k}\right)_{\infty}$. Let $X_{n}=$ $\operatorname{MAX}_{n}\left(\left(\sum_{k=N+1}^{n} \mathbf{M}_{k}\right)_{\infty}\right)$ (see [13] or [11] for the definition and properties 
of the functor $\mathrm{MAX}_{n}$ ), and set, for $x \in X \otimes \mathbf{K}_{0}$,

$$
\|x\|=\sup _{n}\left\|\left(P_{n} \otimes I_{\mathbf{K}}\right) x\right\|_{X_{n} \otimes \mathbf{K}}
$$

It is easy to notice that the Banach space structure of $X$ is as described above. Denote by $Y$ the space $\left(\bigoplus_{n>N} \mathbf{M}_{n}\right)_{c_{0}}$, with the operator space structure inherited from $X$.

For further use, we state the following easy consequence of (3.1).

Lemma 3.2. Suppose $x$ is an element of $X \otimes \mathbf{M}_{m}(m \in \mathbb{N})$. Write $x=$ $\left(x_{i}\right)_{i>N}$ with $x_{i} \in \mathbf{M}_{i} \otimes \mathbf{M}_{m}$. Then

$$
\|x\|_{X \otimes \mathbf{M}_{m}}=\max \left\{\|x\|_{\left(\sum_{n>N} \mathbf{M}_{n}\right)_{c} \otimes \mathbf{M}_{m}}, \max _{N<n \leq m}\left\|\left(P_{n} \otimes I_{\mathbf{M}_{m}}\right) x\right\|_{X_{n} \otimes \mathbf{M}_{m}}\right\} .
$$

Lemma 3.3. $Y$ is a complete $M$-ideal in $X$.

Proof. For $n>N$, define the map $T_{n}: X \rightarrow Y$ by setting

$$
T_{n}\left(\left(x_{i}\right)_{i>N}\right)=\left(x_{N+1}, \ldots, x_{n}, 0,0, \ldots\right) .
$$

We need to show that the sequence $\left(T_{n}\right)$ is an $M$-complete approximate identity (see Definition 1.1 of [1]). Clearly, $T_{n} y \rightarrow y$ for any $y \in Y$. Moreover, suppose $x=\left(x_{i}\right)$ and $z=\left(z_{i}\right)$ are elements of $X \otimes \mathbf{M}_{m}$. By Lemma 3.2, for $n \geq m$,

$$
\begin{gathered}
\left\|\left(T_{n} \otimes I_{\mathbf{M}_{m}}\right)(x)+\left(\left(I-T_{n}\right) \otimes I_{\mathbf{M}_{m}}\right)(z)\right\|=\left\|\left(x_{N+1}, \ldots, x_{n}, z_{n+1}, z_{n+2}, \ldots\right)\right\| \\
=\max \left\{\left\|\left(x_{N+1}, \ldots, x_{n}, z_{n+1}, z_{n+2}, \ldots\right)\right\|_{\left(\sum_{n>N} \mathbf{M}_{n}\right)_{c} \otimes \mathbf{M}_{m}},\right. \\
\left.\max _{N<k \leq m}\left\|\left(x_{N+1}, \ldots, x_{k}\right)\right\|_{X_{k} \otimes \mathbf{M}_{m}}\right\} \\
\quad=\max \left\{\max _{N<k \leq n}\left\|x_{k}\right\|, \sup _{k>n}\left\|z_{k}\right\|, \max _{N<k \leq m}\left\|\left(x_{N+1}, \ldots, x_{k}\right)\right\|_{X_{k} \otimes \mathbf{M}_{m}}\right\} \\
\leq \max \{\|x\|,\|z\|\} .
\end{gathered}
$$

Thus, $\left(T_{n}\right)$ is indeed an $M$-completely approximate identity, and therefore, by Theorem 1.1 of [1], $Y$ is a complete $M$-ideal in $X$.

Lemma 3.4. The quotient $X / Y$ is completely isometric to $\mathbf{K}$.

Proof. Define the map $U: X / Y \rightarrow \mathbf{K}$ by setting $U\left(\left[\left(x_{i}\right)_{i>N}\right]\right)=\lim _{i} x_{i}$. To show that it is a complete isometry, fix $m \in \mathbb{N}$, and consider $x=\left(x_{i}\right)_{i>N}$ $\in X \otimes \mathbf{M}_{m}$. By Lemma 3.2, $\|[x]\|_{X / Y \otimes \mathbf{M}_{m}}=\lim _{i}\left\|x_{i}\right\|=\|U[x]\|_{\mathbf{K} \otimes \mathbf{M}_{m}}$.

Conclusion of the proof of Theorem 3.1. Part (1) of the theorem follows from Lemma 3.4. Suppose $E$ is a finite-dimensional operator space as in part (2), $u: E \rightarrow X$ is a complete contraction, $F=u(E)$, and $\left\|u^{-1}\right\|_{\mathrm{cb}}<C$. Let $F_{n}=P_{n}(F)$ be a subspace of $X_{n}$ (here, $P_{n}$ and $X_{n}$ are as in (3.1)), and let $u_{n}=P_{n} u$. By the definition of $\operatorname{MAX}_{n}$,

$$
\left\|\left(x_{N+1}, \ldots, x_{n}\right)\right\|_{X_{n} \otimes \mathbf{M}_{N}}=\max _{N<k \leq n}\left\|x_{k}\right\|,
$$


hence there exists $n>N$ such that $\left\|\left(u_{n} \otimes I_{\mathbf{M}_{N}}\right) e\right\|>C^{-1}\|e\|$ for any $e \in E \otimes \mathbf{M}_{N}$. By Smith's lemma, $\left\|u_{n}^{-1}\right\|_{\mathrm{cb}}<C\left\|u_{n}^{-1} \otimes I_{\mathbf{M}_{N}}\right\|<C^{2}$.

Since $X_{n}^{*}$ is 1-exact, by [17] there exist operators $v: E \rightarrow \mathbf{R} \oplus \mathbf{C}$ and $w: \mathbf{R} \oplus \mathbf{C} \rightarrow X_{n}$ so that $\|v\|_{\mathrm{cb}}\|w\|_{\mathrm{cb}} \leq 4 \sqrt{2} C$ and $u_{n}=w v$. Let $G=v(E)$. Then $d_{\mathrm{cb}}(E, G) \leq\|v\|_{\mathrm{cb}}\left\|u_{n}^{-1} w\right\|_{\mathrm{cb}}<4 \sqrt{2} C^{3}$.

To prove the last assertion, note that, by the reasoning above and Proposition 2.5, any $N$-dimensional $C+$-BDR space $E$ embeds into $\mathbf{R}_{N} \oplus \mathbf{C}_{N}$ $4 \sqrt{2}(C+\varepsilon)^{3}$-completely isomorphically for any $\varepsilon>0$. Letting $\varepsilon$ approach 0 , and applying a classical compactness argument, we complete the proof.

4. Proof of Theorem 1.3(2): $E$ is $\mathbf{R}_{m} \oplus \mathbf{C}_{n}$. In this section, we complete the proof of Theorem 1.3(2). For the convenience of working with Hilbert spaces as much as possible, we use the sum $\oplus_{2}$ : if $X$ and $Y$ are operator spaces, and $x$ and $y$ are elements of $X \otimes \mathbf{K}_{0}$ and $Y \otimes \mathbf{K}_{0}$, respectively, define

$$
\|x \oplus y\|_{\left(X \oplus_{2} Y\right) \otimes \mathbf{K}}=\max \left\{\|x\|,\|y\|,\|x \oplus y\|_{\operatorname{MIN}\left(X \oplus_{2} Y\right) \otimes \mathbf{K}}\right\} .
$$

Clearly, Ruan's axioms are satisfied, so $X \oplus_{2} Y$ is indeed an operator space.

Note that any $N$-dimensional subspace $E$ of $\mathbf{R} \oplus_{2} \mathbf{C}$ is contained in $\mathbf{R}_{N} \oplus_{2} \mathbf{C}_{N}$. In view of Theorem 3.1, the proof of Theorem 1.3(2) follows from:

TheOREM 4.1. Suppose $E$ is an $N$-dimensional subspace of $\mathbf{R}_{N} \oplus_{2} \mathbf{C}_{N}$ which is $\lambda$-BDR. Then $d_{\mathrm{cb}}\left(E, \mathbf{R}_{m} \oplus_{2} \mathbf{C}_{n}\right) \leq 32 \sqrt{2} \lambda$ for suitable $m, n \in \mathbb{Z}_{+}$.

Denote by $A_{\mathbf{R}}$ and $A_{\mathbf{C}}$ the orthogonal projections from $E$ on $\mathbf{R}_{N}$ and $\mathbf{C}_{N}$, respectively. By polar decomposition, there exists an orthonormal basis $\left(e_{i}\right)_{i=1}^{N}$ in $E$ such that $A_{\mathbf{R}} e_{i}=a_{i}^{(\mathbf{R})} e_{i}^{(\mathbf{R})}$, with $0 \leq a_{N}^{(\mathbf{R})} \leq \cdots \leq a_{1}^{(\mathbf{R})} \leq 1$ and $e_{i}^{(\mathbf{R})}$ being an orthonormal basis for $\mathbf{R}_{N}$. Then, for $1 \leq i \leq N, A_{\mathbf{C}} e_{i}=$ $a_{i}^{(\mathbf{C})} e_{i}^{(\mathbf{C})}$, where $e_{i}^{(\mathbf{C})}$ is a unit vector and $a_{i}^{(\mathbf{C})}=\sqrt{1-\left(a_{i}^{(\mathbf{R})}\right)^{2}}$. Note that, for $i \neq j$,

$$
\left\langle e_{i}, e_{j}^{(\mathbf{R})}\right\rangle=\left\langle a_{i}^{(\mathbf{R})} e_{i}^{(\mathbf{R})}+a_{i}^{(\mathbf{C})} e_{i}^{(\mathbf{C})}, e_{j}^{(\mathbf{R})}\right\rangle=0 .
$$

Moreover,

$$
\begin{aligned}
0=\left\langle e_{i}, e_{j}\right\rangle & =\left\langle a_{i}^{(\mathbf{R})} e_{i}^{(\mathbf{R})}+a_{i}^{(\mathbf{C})} e_{i}^{(\mathbf{C})}, a_{j}^{(\mathbf{R})} e_{j}^{(\mathbf{R})}+a_{j}^{(\mathbf{C})} e_{j}^{(\mathbf{C})}\right\rangle \\
& =a_{i}^{(\mathbf{C})} a_{j}^{(\mathbf{C})}\left\langle e_{i}^{(\mathbf{C})}, e_{j}^{(\mathbf{C})}\right\rangle,
\end{aligned}
$$

and therefore, the vectors $\left(e_{i}^{(\mathbf{C})}\right)_{i=1}^{N}$ form an orthonormal basis in $\mathbf{C}_{N}$ (certain minor changes to this construction need to be made if $a_{i}^{(\mathbf{C})}=0$ for some $i$ 's). One can also show that $\left\langle e_{i}, e_{j}^{(\mathbf{C})}\right\rangle=0$ for $i \neq j$.

LEMMA 4.2. $\left(e_{i}\right)_{i=1}^{N}$ is a 1-completely unconditional basis in $E$. 
Proof. Suppose $\lambda_{1}, \ldots, \lambda_{N}$ are complex numbers of absolute value not exceeding 1 . We have to show that the operator $\Lambda=\operatorname{diag}\left(\left(\lambda_{i}\right)_{i=1}^{N}\right)$ is completely contractive. To this end, consider an operator $\widetilde{\Lambda}$ on $\mathbf{R}_{N} \oplus_{2} \mathbf{C}_{N}$ mapping $e_{i}^{(\mathbf{R})}$ (or $e_{i}^{(\mathbf{C})}$ ) into $\lambda_{i} e_{i}^{(\mathbf{R})}$ (resp. $\lambda_{i} e_{i}^{(\mathbf{C})}$ ) for $1 \leq i \leq N$. By the discussion preceding the statement of this lemma, the restrictions of $\widetilde{\Lambda}$ to $\mathbf{R}_{N}$ and $\mathbf{C}_{N}$ are contractive, hence completely contractive (row and column spaces are 1-homogeneous). Thus, by the homogeneity of minimal spaces, and by (4.1), $\widetilde{\Lambda}$ is completely contractive. To complete the proof, observe that the restriction of $\widetilde{\Lambda}$ to $E$ coincides with $\Lambda$.

This lemma, together with (4.1), yields:

Corollary 4.3. Suppose $\mathcal{I}$ is a subset of $\{1, \ldots, N\}$. Let $E_{\mathcal{I}}=$ $\operatorname{span}\left[e_{i} \mid i \in \mathcal{I}\right]$, and $E_{\mathcal{I}}^{\perp}=\operatorname{span}\left[e_{i} \mid i \notin \mathcal{I}\right]$. Then the formal identity map id $: E \rightarrow E_{\mathcal{I}} \oplus_{2} E_{\mathcal{I}}^{\perp}$ is completely contractive, and $\left\|\mathrm{id}^{-1}\right\|_{\mathrm{cb}} \leq \sqrt{2}$.

Proof. For simplicity, denote the space $E_{\mathcal{I}} \oplus_{2} E_{\mathcal{I}}^{\perp}$ by $F$. Consider $x=$ $\sum_{i} e_{i} \otimes x_{i} \in E \otimes \mathbf{K}$. Then

$$
\begin{array}{r}
\|x\|_{E \otimes \mathbf{K}}=\max \left\{\left\|\sum_{i=1}^{N}\left(a_{i}^{(\mathbf{R})}\right)^{2} x_{i}^{*} x_{i}\right\|^{1 / 2},\left\|\sum_{i=1}^{N}\left(a_{i}^{(\mathbf{C})}\right)^{2} x_{i} x_{i}^{*}\right\|^{1 / 2},\right. \\
\left.\left\|\sum_{i=1}^{N} e_{i} \otimes x_{i}\right\|_{\operatorname{MIN}\left(\ell_{2}^{N}\right) \otimes \mathbf{K}}\right\},
\end{array}
$$

while

$$
\begin{aligned}
& \|x\|_{F \otimes \mathbf{K}}=\max \left\{\left\|\sum_{i \in \mathcal{I}}\left(a_{i}^{(\mathbf{R})}\right)^{2} x_{i}^{*} x_{i}\right\|^{1 / 2},\left\|\sum_{i \in \mathcal{I}^{c}}\left(a_{i}^{(\mathbf{R})}\right)^{2} x_{i}^{*} x_{i}\right\|^{1 / 2},\right. \\
& \left.\left\|\sum_{i \in \mathcal{I}}\left(a_{i}^{(\mathbf{C})}\right)^{2} x_{i} x_{i}^{*}\right\|^{1 / 2},\left\|\sum_{i \in \mathcal{I}^{c}}\left(a_{i}^{(\mathbf{C})}\right)^{2} x_{i} x_{i}^{*}\right\|^{1 / 2},\left\|\sum_{i=1}^{N} e_{i} \otimes x_{i}\right\|_{\operatorname{MiN}\left(\ell_{2}^{N}\right) \otimes \mathbf{K}}\right\} .
\end{aligned}
$$

Comparing the two displayed expressions yields the result.

Turning back to the proof of Theorem 4.1, denote by $m$ the largest number $i$ for which $a_{i}^{(\mathbf{R})} \geq 1 / \sqrt{2}$ (if $a_{1}^{(\mathbf{R})}<1 / \sqrt{2}$, set $m=0$ ), and let $n=N-m$. Let $E_{\mathbf{R}}=\operatorname{span}\left[e_{i} \mid 1 \leq i \leq m\right], E_{\mathbf{C}}=\operatorname{span}\left[e_{i} \mid m<i \leq N\right]$. For a compact operator $T \in B(H, K)$ ( $H$ and $K$ are Hilbert spaces), we denote by $\|T\|_{2}$ its Hilbert-Schmidt norm. That is, $\|T\|_{2}=\left(\sum_{n} t_{n}^{2}\right)^{1 / 2}$, where $t_{1} \geq t_{2} \geq$ $\cdots \geq 0$ are the singular numbers of $T$. Equivalently, $\|T\|_{2}^{2}=\sum_{i, j}\left|\left\langle T e_{i}, f_{j}\right\rangle\right|^{2}$, where $\left(e_{i}\right)$ and $\left(f_{j}\right)$ are orthonormal bases in $H$ and $K$, respectively.

To complete the proof, it suffices to show that

$$
\max \left\{\left\|\left.A_{\mathbf{C}}\right|_{E_{\mathbf{R}}}\right\|_{2},\left\|\left.A_{\mathbf{R}}\right|_{E_{\mathbf{C}}}\right\|_{2}\right\} \leq 16 \sqrt{2} \lambda .
$$


Indeed, this would imply that $E_{\mathbf{R}}$ and $E_{\mathbf{C}}$ are $32 \lambda$-completely isomorphic to $\mathbf{R}_{m}$ and $\mathbf{C}_{n}$, respectively. An application of Corollary 4.3 would then yield the result. Thus, it remains to prove:

Proposition 4.4. In the above notation, $\left\|\left.A_{\mathbf{C}}\right|_{E_{\mathbf{R}}}\right\|_{2} \leq 16 \sqrt{2} \lambda$.

Proof. If $m=0$, there is nothing to prove. If $m \geq 1$, denote $\left.A_{\mathbf{C}}\right|_{E_{\mathbf{R}}}$ by $A$ for simplicity of notation. Let

$$
X_{1}=\left(\sum_{n>N} \operatorname{MAX}_{n}\left(E_{\mathbf{R}}\right)\right)_{c}, \quad Y=\left(\sum_{n>N} \operatorname{MAX}_{n}\left(E_{\mathbf{R}}\right)\right)_{c_{0}}, \quad X=X_{1} \oplus_{2} E_{\mathbf{C}}
$$

By Proposition 3.2 of [1], $Y$ is a complete $M$-ideal in $X_{1}$. Imitating the proof of Lemma 3.4, one can show that $X_{1} / Y=E_{\mathbf{R}}$ completely isometrically. Therefore, $X_{1}^{* *}$ contains $E_{\mathbf{R}}$ as a complete $M$-summand. Finally, $\mathbf{M}_{s}\left(X^{* *}\right)=$ $\left(\mathbf{M}_{s}(X)\right)^{* *}$ for any $s \in \mathbb{N}$, hence $X^{* *}=X_{1}^{* *} \oplus_{2} E_{\mathbf{C}}$, and therefore, $X^{* *}$ contains $E \sqrt{2}$-completely isomorphically. By Corollary 2.6, $X$ contains $E$ $\sqrt{2} \lambda+$-ci.

Pick $C>\lambda$, and consider a complete contraction $u: E \rightarrow X$ satisfying $\left\|u^{-1}\right\|_{\mathrm{cb}} \leq \sqrt{2} C$. Denote the "natural truncation" of $u$ to $E_{\mathbf{C}}$ (or the $n$th summand of $X_{1}, n>N$ ) by $u_{0}$ (respectively, $u_{n}$ ). More precisely, we view $u_{0}$ (resp. $\left.u_{n}\right)$ as a map from $E$ to $E_{\mathbf{C}}\left(\right.$ resp. $\left.\operatorname{MAX}_{n}\left(E_{\mathbf{R}}\right)\right)$. In this notation, $F=\operatorname{ker} u_{0}$ is an $M$-dimensional subspace of $E(M \geq m)$. Let $v$ be an isometry from $\mathbf{R}_{M}$ onto $F$. To complete the proof, it suffices to show that

(1) $\|v\|_{\mathrm{cb}} \geq \max \left\{1,\|A\|_{2} / 2\right\}$,

(2) $\left\|u_{n} v\right\|_{\mathrm{cb}} \leq 8$ for any $n>N$.

Indeed, then $\|u v\|_{\mathrm{cb}}=\sup _{n}\left\|u_{n} v\right\|_{\mathrm{cb}} \leq 8$. On the other hand, $v=u^{-1} \circ(u v)$, hence the above inequalities imply

$$
\|A\|_{2} / 2 \leq\|v\|_{\mathrm{cb}}<\sqrt{2} C\|u v\|_{\mathrm{cb}} \leq 8 \sqrt{2} C .
$$

Since $C>\lambda$ is arbitrary, we conclude that $\left\|A_{2}\right\|_{2} \leq 16 \sqrt{2} \lambda$.

We start by proving (4.2(1)). Denote by $Q$ and $Q^{\perp}$ the orthogonal projections from $F$ onto $E_{\mathbf{R}}$ and $E_{\mathbf{C}}$, respectively. Reasoning as in the proof of Lemma 4.2 (see also the discussion preceding it), we can find an orthonormal basis $\left(f_{i}\right)_{i=1}^{M}$ in $F$ such that $\left\langle Q f_{i}, Q f_{j}\right\rangle=\left\langle Q^{\perp} f_{i}, Q^{\perp} f_{j}\right\rangle=0$ if $i \neq j$. By changing the numbering if necessary, assume that $\left\|Q^{\perp} f_{i}\right\| \geq 1 / \sqrt{2}$ for $1 \leq i \leq l$, and $\left\|Q f_{i}\right\|>1 / \sqrt{2}$ for $l<i \leq M$. Let $F_{1}=\operatorname{span}\left[f_{i} \mid 1 \leq i \leq l\right]$, $F_{2}=\operatorname{span}\left[f_{i} \mid l<i \leq M\right]$, and $G_{s}=v^{-1}\left(F_{s}\right)$ for $s=1,2$. We can identify $G_{1}$ and $G_{2}$ with $\mathbf{R}_{l}$ and $\mathbf{R}_{M-l}$, respectively. Note that

$$
\|v\|_{\mathrm{cb}} \geq \max \left\{\left\|\left.v\right|_{G_{1}}\right\|_{\mathrm{cb}},\left\|\left.v\right|_{G_{2}}\right\|_{\mathrm{cb}}\right\} \geq\left(\left\|\left.v\right|_{G_{1}}\right\|_{\mathrm{cb}}^{2}+\left\|\left.v\right|_{G_{2}}\right\|_{\mathrm{cb}}^{2}\right)^{1 / 2} / \sqrt{2} .
$$

However,

$$
\left\|\left.v\right|_{G_{1}}\right\|_{\mathrm{cb}} \geq\left\|\left.Q^{\perp} v\right|_{G_{1}}\right\|_{C B\left(\mathbf{R}_{l}, \mathbf{C}_{M}\right)}=\left\|\left.Q^{\perp} v\right|_{G_{1}}\right\|_{2} \geq \sqrt{l / 2} .
$$


Similarly,

$$
\left\|\left.v\right|_{G_{2}}\right\|_{\mathrm{cb}} \geq\left\|\left.A Q v\right|_{G_{2}}\right\|_{C B\left(\mathbf{R}_{M-l}, \mathbf{C}_{M}\right)}=\left\|\left.A Q v\right|_{G_{2}}\right\|_{2}=\left(\sum_{i=l+1}^{M}\left\|A Q f_{i}\right\|^{2}\right)^{1 / 2} .
$$

To evaluate $\|A\|_{2}$, introduce the vectors $f_{i}^{\prime} \in E_{\mathbf{R}}(1 \leq i \leq M)$ in such a way that $E_{\mathbf{R}}=\operatorname{span}\left[f_{i}^{\prime} \mid 1 \leq i \leq M\right]$, and, for each $i,\left\|f_{i}^{\prime}\right\|$ equals 0 or 1 , and $f_{i}^{\prime}=Q f_{i} /\left\|Q f_{i}\right\|$ provided $Q f_{i} \neq 0$ (this is possible, since $M \geq m=\operatorname{dim} E_{\mathbf{R}}$ ). Since $A$ is a contraction, we have

$\|A\|_{2}^{2}=\sum_{i=1}^{M}\left\|A f_{i}^{\prime}\right\|^{2} \leq l+2 \sum_{i=l+1}^{M}\left\|A Q f_{i}\right\|^{2} \leq 2\left(\left\|\left.v\right|_{G_{1}}\right\|_{\mathrm{cb}}^{2}+\left\|\left.v\right|_{G_{2}}\right\|_{\mathrm{cb}}^{2}\right) \leq 4\|v\|_{\mathrm{cb}}^{2}$.

Moreover, $v$ is an isometry, thus we obtain (4.2(1)).

Next we tackle (4.2(2)). Fix $n$. By [17], there exist operators $T_{\mathbf{R}}: F \rightarrow$ $\mathbf{R}_{M}, T_{\mathbf{C}}: F \rightarrow \mathbf{C}_{M}, S_{\mathbf{R}}: \mathbf{R}_{M} \rightarrow \operatorname{MAX}_{n}\left(E_{\mathbf{R}}\right)$, and $S_{\mathbf{C}}: \mathbf{C}_{M} \rightarrow \operatorname{MAX}_{n}\left(E_{\mathbf{R}}\right)$ so that $\left.u_{n}\right|_{F}=S_{\mathbf{R}} T_{\mathbf{R}}+S_{\mathbf{C}} T_{\mathbf{C}}$ and $\max \left\{\left\|T_{\mathbf{R}}\right\|_{\mathrm{cb}}\left\|S_{\mathbf{R}}\right\|_{\mathrm{cb}},\left\|T_{\mathbf{C}}\right\|_{\mathrm{cb}}\left\|S_{\mathbf{C}}\right\|_{\mathrm{cb}}\right\} \leq$ $2 \sqrt{2}$. Then

$$
\left\|S_{\mathbf{R}} T_{\mathbf{R}} v\right\|_{\mathrm{cb}} \leq\left\|S_{\mathbf{R}}\right\|_{\mathrm{cb}}\left\|T_{\mathbf{R}} v\right\|_{\mathrm{cb}}=\left\|S_{\mathbf{R}}\right\|_{\mathrm{cb}}\left\|T_{\mathbf{R}} v\right\| \leq 2 \sqrt{2}
$$

Moreover,

$$
\left\|S_{\mathbf{C}}\right\|_{\mathrm{cb}} \geq\left\|A_{\mathbf{R}} S_{\mathbf{C}}\right\|_{C B\left(\mathbf{C}_{M}, \mathbf{R}_{N}\right)}=\left\|A_{\mathbf{R}} S_{\mathbf{C}}\right\|_{2} \geq\left\|S_{\mathbf{C}}\right\|_{2} / \sqrt{2},
$$

hence

$$
\left\|S_{\mathbf{C}} T_{\mathbf{C}} v\right\|_{\mathrm{cb}} \leq\left\|S_{\mathbf{C}} T_{\mathbf{C}} v\right\|_{2} \leq\left\|S_{\mathbf{C}}\right\|_{2}\left\|T_{\mathbf{C}} v\right\| \leq 4
$$

Thus,

$$
\left\|u_{n} v\right\|_{\mathrm{cb}} \leq\left\|S_{\mathbf{R}} T_{\mathbf{R}} v\right\|_{\mathrm{cb}}+\left\|S_{\mathbf{C}} T_{\mathbf{C}} v\right\|_{\mathrm{cb}} \leq 8 .
$$

This establishes $(4.2(2))$.

Proof of Theorem 1.3(2). Suppose an $N$-dimensional space $E$ is $\lambda+$ BDR. Pick $C>\lambda$. By Theorem 3.1, there exists a subspace $F$ of $\mathbf{C}_{N} \oplus_{2} \mathbf{R}_{N}$ such that $d_{\mathrm{cb}}(E, F)<8 C^{3}$. By Corollary 2.6 and Proposition 2.5, $F$ is $2^{6} C^{8}$-BDR. By Theorem 4.1, $F$ is $2^{23 / 2} C^{8}$-ci to $G_{C}=\mathbf{R}_{m(C)} \oplus_{2} \mathbf{C}_{n(C)}$. Thus, $d_{\mathrm{cb}}\left(E, G_{C}\right) \leq 2^{29 / 2} C^{11}$. Find a sequence $\left(C_{j}\right)$, decreasing to $\lambda$, such that $m=m\left(C_{j}\right)$ for any $j$ (then $n=N-m\left(C_{j}\right)=n\left(C_{j}\right)$ ). Clearly, we have $d_{\mathrm{cb}}\left(E, \mathbf{R}_{m} \oplus_{\infty} \mathbf{C}_{n}\right) \leq 2^{15} \lambda^{10}$.

5. Proof of Theorem 1.4, and similar lower estimates. This section is devoted to the proof of Theorem 1.4. Clearly, $\mathbb{C}$ is 1-BDR. A series of lemmas helps us rule out other spaces. The first lemma seems to be partly folklore. 


\section{LEMMA 5.1.}

(1) Suppose $\left(E_{k}\right)_{k=1}^{\infty}$ is a sequence of Banach spaces, and $E$ is a finitedimensional subspace of $\left(\sum_{k} E_{k}\right)_{c_{0}}$. Then there exists $N \in \mathbb{N}$ such that $E$ is isometric to a finite-dimensional subspace of $\left(\sum_{k=1}^{N} E_{k}\right)_{\infty}$.

(2) Suppose $\left(E_{k}\right)_{k=1}^{\infty}$ is a sequence of operator spaces, and $E$ is a finitedimensional subspace of $\left(\sum_{k} E_{k}\right)_{c_{0}}$. Then there exists $N \in \mathbb{N}$ such that $E$ is completely isometric to a finite-dimensional subspace of $\left(\sum_{k=1}^{N} E_{k}\right)_{\infty}$

Proof. We prove part (2), since (1) can be dealt with in a similar manner. Let $n=\operatorname{dim} E$. Suppose $\left(e_{i}\right)_{i=1}^{n}$ is an Auerbach basis in $E$ - that is, $\max _{i}\left|\alpha_{i}\right| \leq\left\|\sum_{i} \alpha_{i} e_{i}\right\| \leq \sum_{i}\left|\alpha_{i}\right|$ for each sequence $\left(\alpha_{i}\right)$ of scalars. In particular, the projection $R_{j}: E \rightarrow E: \sum_{i} \alpha_{i} e_{i} \mapsto \alpha_{j} e_{j}$ is contractive for every $j$. Since it is a rank one projection, it must also be completely contractive. Thus, for any $\left(a_{i}\right)_{i=1}^{n} \subset \mathbf{K},\left\|\sum_{i} a_{i} \otimes e_{i}\right\| \geq \max _{i}\left\|a_{i}\right\|$.

Now write $e_{i}=\left(e_{i k}\right)_{k=1}^{\infty}$ with $e_{i k} \in E_{k}$. There exists $N \in \mathbb{N}$ such that $\left\|e_{i k}\right\|<1 / n$ for any $1 \leq i \leq n$, and any $k>N$. For such $k$, and for any $\left(a_{i}\right)_{i=1}^{n} \subset \mathbf{K},\left\|\sum_{i} a_{i} \otimes e_{i k}\right\|<\max _{i}\left\|a_{i}\right\|$. Therefore,

$$
\left\|\sum_{i} a_{i} \otimes e_{i}\right\|=\sup _{k}\left\|\sum_{i} a_{i} \otimes e_{i k}\right\|=\max _{1 \leq k \leq N}\left\|\sum_{i} a_{i} \otimes e_{i k}\right\|,
$$

which implies that $E$ is completely isometric to a subspace of $\left(\sum E_{k}\right)_{k=1}^{N}$, spanned by the vectors $\widetilde{e}_{i}=\left(e_{i k}\right)_{k=1}^{N}(1 \leq i \leq n)$.

We next apply this lemma to our situation.

LEMMA 5.2. Suppose a finite-dimensional operator space $E$ is 1-BDR. Then, for some $N \in \mathbb{N}, E$ embeds into $\ell_{\infty}^{N}$ isometrically, and into $\mathbf{M}_{N}$ completely isometrically.

Proof. For $n \in \mathbb{N}$, find $e_{1 n}^{*}, \ldots, e_{M_{n} n}^{*} \in E^{*}$ of norm 1 so that, for any $e \in E$,

$$
\max _{1 \leq k \leq M_{n}}\left|\left\langle e_{k n}^{*}, e\right\rangle\right| \geq\left(1-2^{-n}\right)\|e\|
$$

Define the operator space $E_{n}$ by setting, for $e \in E \otimes \mathbf{K}_{0}$,

$$
\|e\|_{E_{n} \otimes \mathbf{K}}=\max \left\{\left(1-2^{-n}\right)\|e\|_{E \otimes \mathbf{K}}, \max _{1 \leq k \leq M_{n}}\left\|\left(e_{k n}^{*} \otimes I_{\mathbf{K}}\right) e\right\|_{\mathbf{K}}\right\} .
$$

Note that, for $e \in E$,

$$
\|e\|_{E_{n}}=\max _{1 \leq k \leq M_{n}}\left|\left\langle e_{k n}^{*}, e\right\rangle\right| .
$$

Let $X=\left(\sum_{n=1}^{\infty} E_{n}\right)_{c_{0}}$. Then the map

$$
u: E \rightarrow X^{* *}=\left(\sum_{n=1}^{\infty} E_{n}\right)_{\ell_{\infty}}: e \mapsto(e, e, \ldots)
$$


is a complete isometry. On the other hand, if $E$ embeds into $X$ isometrically, then, by Lemma 5.1 , it must embed into $\left(\sum_{n=1}^{K} E_{n}\right)_{\infty}$ for some $K \in \mathbb{N}$. By (5.1), $E_{n}$ embeds isometrically into $\ell_{\infty}^{M_{n}}$. Therefore, $E$ embeds isometrically into $\ell_{\infty}^{N}$ for $N=\sum_{n=1}^{K} M_{n}$.

Next test 1-bidual representability with $X=\left(\sum_{k=1}^{\infty} \mathbf{M}_{k}\right)_{c_{0}}$. The space $X^{* *}=\left(\sum_{k=1}^{\infty} \mathbf{M}_{k}\right)_{\infty}$ contains $\mathbf{B}$, hence it also contains $E$. Therefore, $X$ contains $E$. By Lemma 5.1, $E$ embeds into $\left(\sum_{k=1}^{M} \mathbf{M}_{k}\right)_{\infty}$ for some $M$. A fortiori, $E$ embeds completely isometrically into $\mathbf{M}_{N}$ with $N=M(M+1) / 2$.

Corollary 5.3. If a 1-BDR space $E$ has dimension at least 2 , then $E$ is not strictly convex.

Recall that a Banach space $E$ is called strictly convex if, for any $e_{1}, e_{2}$ in $E$, the equality $\left\|e_{1}+e_{2}\right\|^{2}=2\left(\left\|e_{1}\right\|^{2}+\left\|e_{2}\right\|^{2}\right)$ implies $e_{1}=e_{2}$.

Proof. By Lemma 5.2, there exist linear functionals $f_{1}, \ldots, f_{N} \in E^{*}$ such that $\|e\|=\max _{1 \leq i \leq N}\left|\left\langle f_{i}, e\right\rangle\right|$ for any $e \in E$. Therefore, there exist $i$ and two distinct elements $e_{1}, e_{2}$ of the unit ball of $E$ satisfying

$$
\left\|e_{1}\right\|=\left\|e_{2}\right\|=\left|\left\langle f_{i}, e_{1}\right\rangle\right|=\left|\left\langle f_{i}, e_{2}\right\rangle\right|=1 .
$$

Then $4=\left\|e_{1}+e_{2}\right\|^{2}=2\left(\left\|e_{1}\right\|^{2}+\left\|e_{2}\right\|^{2}\right)$.

Proof of Theorem 1.4. Suppose, for the sake of contradiction, that $\operatorname{dim} E$ $>1$, and $E$ is 1-BDR. By Lemma 5.2, $E$ embeds into $\mathbf{M}_{N}$ completely isometrically. We then construct an operator space $X$, isomorphic to $c_{0}\left(\mathbf{M}_{N}\right)$, which is strictly convex (hence, by Corollary $5.3, X$ cannot contain $E$ isometrically), but such that $X^{* *}$ contains $\mathbf{M}_{N}$ completely isometrically.

To this end, find a dense sequence $\left(f_{j}\right)_{j=1}^{\infty}$ in the unit ball of $\mathbf{M}_{N}^{*}$. For $x=\left(x_{1}, x_{2}, \ldots\right) \in c_{0}\left(\mathbf{M}_{N}\right)$, set

$$
\begin{aligned}
\|x\|_{X} & =\sup _{n}\|x\|_{n}, \quad \text { where } \\
\|x\|_{n} & =\left(\left\|x_{n}\right\|^{2}+\sum_{j, k=1}^{\infty} 10^{-(n+j+k)}\left|\left\langle f_{j}, x_{k}\right\rangle\right|^{2}\right)^{1 / 2} .
\end{aligned}
$$

Observe that

$$
\begin{aligned}
\sum_{j, k=1}^{\infty} 10^{-(n+j+k)}\left|\left\langle f_{j}, x_{k}\right\rangle\right|^{2} & \leq 10^{-(n+1)} \sum_{j, k=1}^{\infty} 10^{-(j+k-1)}=10^{-(n+1)} \sum_{\ell=0}^{\infty} l 10^{-l} \\
& =10^{-(n+1)}\left(\frac{1}{1-1 / 10}\right)^{2}<1.25 \cdot 10^{-(n+1)},
\end{aligned}
$$

SO

$$
\|x\|_{n}<\left\|x_{n}\right\|+10^{-n}\|x\|_{c_{0}\left(\mathbf{M}_{N}\right)}
$$


hence $\|\cdot\|_{X}$ is well defined. To show that $\|\cdot\|_{n}$ is a norm, note that $\|x\|_{n}=$ $\left\|J_{n} x\right\|_{\mathbf{M}_{N} \oplus_{2} \ell_{2}(\mathbb{N} \times \mathbb{N})}$, where

$$
J_{n}: c_{0}\left(\mathbf{M}_{N}\right) \rightarrow \mathbf{M}_{N} \oplus_{2} \ell_{2}(\mathbb{N} \times \mathbb{N}): x \mapsto\left(x_{n},\left(10^{-(n+j+k)}\left\langle f_{j}, x_{k}\right\rangle\right)_{j, k}\right) .
$$

By (5.3), $\lim _{n}\left\|J_{n} x\right\|=0$ for any $x$, hence (5.2) describes $X$ as a subspace of $\left(\sum\left(\mathbf{M}_{N} \oplus_{2} \ell_{2}\right)\right)_{c_{0}}$, and $\|\cdot\|_{X}$ is a norm. Thus, $X^{* *}$ is isomorphic to $\ell_{\infty}\left(\mathbf{M}_{N}\right)$, and the norm is also given by (5.2).

Finally, note that the supremum in (5.2) is attained. Indeed, if $\|x\|_{c_{0}\left(\mathbf{M}_{N}\right)}=1$, find $K \in \mathbb{N}$ such that $\left\|x_{n}\right\|^{2}+10^{-n}<1 / 4$ for $n \geq K$. For such $n,\|x\|_{n}<1 / 2$, while $\|x\|_{X}>1$. Therefore, $\|x\|_{X}=\max _{n<K}\|x\|_{n}$.

To show that $X$ is strictly convex, suppose $\left\|x^{(1)}+x^{(2)}\right\|^{2}=2\left(\left\|x^{(1)}\right\|^{2}+\right.$ $\left\|x^{(2)}\right\|^{2}$ ) for some $x^{(1)}, x^{(2)} \in X$. By the observation above, there exists $n \in \mathbb{N}$ such that $\left\|x^{(1)}+x^{(2)}\right\|=\left\|x^{(1)}+x^{(2)}\right\|_{n}$. Therefore (writing $\left.x^{(s)}=\left(x_{n}^{(s)}\right)_{n=1}^{\infty}\right)$,

$$
\begin{aligned}
0 \geq & 2\left(\left\|x^{(1)}\right\|_{n}^{2}+\left\|x^{(2)}\right\|_{n}^{2}\right)-\left\|x^{(1)}+x^{(2)}\right\|_{n}^{2} \\
= & 2\left(\left\|x_{n}^{(1)}\right\|^{2}+\left\|x_{n}^{(2)}\right\|^{2}\right)-\left\|x_{n}^{(1)}+x_{n}^{(2)}\right\|^{2} \\
& +\sum_{j, k=1}^{\infty} 10^{-(n+j+k)}\left(2\left(\left|\left\langle f_{j}, x_{k}^{(1)}\right\rangle\right|^{2}+\left|\left\langle f_{j}, x_{k}^{(2)}\right\rangle\right|^{2}\right)-\left|\left\langle f_{j}, x_{k}^{(1)}+x_{k}^{(2)}\right\rangle\right|^{2}\right) .
\end{aligned}
$$

But, by the triangle inequality,

$$
2\left(\left\|x_{n}^{(1)}\right\|^{2}+\left\|x_{n}^{(2)}\right\|^{2}\right) \geq\left\|x_{n}^{(1)}+x_{n}^{(2)}\right\|^{2},
$$

and

$$
2\left(\left|\left\langle f_{j}, x_{k}^{(1)}\right\rangle\right|^{2}+\left|\left\langle f_{j}, x_{k}^{(2)}\right\rangle\right|^{2}\right) \geq\left|\left\langle f_{j}, x_{k}^{(1)}+x_{k}^{(2)}\right\rangle\right|^{2} .
$$

In the last display, equality holds if and only if $x_{k}^{(1)}=x_{k}^{(2)}$ for every $k$. Thus, $x^{(1)}=x^{(2)}$.

Define the operator space structure on $X$ by setting, for $x \in X \otimes \mathbf{K}_{0}$,

$$
\|x\|=\max \left\{\|x\|_{\operatorname{MIN}(X) \otimes \mathbf{K}_{0}},\|x\|_{c_{0}\left(\mathbf{M}_{N}\right) \otimes \mathbf{K}_{0}}\right\} .
$$

In other words, the operator space structure on $X$ is generated by its "natural" embedding into $\operatorname{MIN}(X) \oplus c_{0}\left(\mathbf{M}_{N}\right)$. Then $X^{* *}$ embeds into $\operatorname{MIN}\left(X^{* *}\right) \oplus$ $\ell_{\infty}\left(\mathbf{M}_{N}\right)$, and, for $x \in X^{* *} \otimes \mathbf{K}_{0}$,

$$
\|x\|=\max \left\{\|x\|_{\operatorname{MIN}\left(X^{* *}\right) \otimes \mathbf{K}_{0}},\|x\|_{\ell_{\infty}\left(\mathbf{M}_{N}\right) \otimes \mathbf{K}_{0}}\right\} .
$$

Define $U: \mathbf{M}_{N} \rightarrow X^{* *}: a \mapsto\left(\left(1-10^{-n}\right) a\right)_{n=1}^{\infty}$. By (5.2) and the discussion following it, and by (5.4), $U$ is a complete contraction. Furthermore, for any $a \in \mathbf{M}_{N} \otimes \mathbf{K}_{0}$,

$$
\left\|\left(U \otimes I_{\mathbf{K}}\right) a\right\|_{X^{* *} \otimes \mathbf{K}} \geq \sup _{n}\left(1-10^{-n}\right)\|a\|_{\mathbf{M}_{N} \otimes \mathbf{K}}=\|a\|,
$$

hence $U$ is a complete isometry.

REMARK 5.4. In a similar way one can prove the commutative version of Theorem 1.4: if a finite-dimensional Banach space $E$ is such that a Banach 
space $X$ contains $E$ isometrically whenever $X^{* *}$ contains $E$ isometrically, then $E$ is 1-dimensional. Indeed, one can imitate the proof of Lemma 5.2 to show that such an $E$ embeds into $\ell_{\infty}^{N}$ for some $N$. Then, as above, one constructs a strictly convex Banach space $Z$ whose dual contains $\ell_{\infty}^{N}$.

Next we investigate the smallest $C$ for which the given space $E$ is $C$-BDR.

TheOREm 5.5. Suppose $E$ is a finite-dimensional $C$-injective operator space which is $\lambda+-B D R$. Then $\lambda \geq \operatorname{ex}\left(E^{*}\right) /(C \operatorname{ex}(E))$.

Proof. Suppose, for the sake of contradiction, that $\lambda<\operatorname{ex}\left(E^{*}\right) /(C \operatorname{ex}(E))$. Pick $C_{1}>\operatorname{ex}(E), C_{2}<\operatorname{ex}\left(E^{*}\right)$, and $C_{3}>\lambda$ such that $C_{2} /\left(C_{1} C_{3}\right)>C$. Following the proof of Theorem 3.1, find $E_{1} \hookrightarrow \mathbf{M}_{N}$ such that $d_{\mathrm{cb}}\left(E, E_{1}\right)<C_{1}$. Also, define the spaces $X_{n}(n>N)$ and $X$ as in the proof of that theorem. We know that $X^{* *}$ contains $\mathbf{B}$ as a complete $M$-summand. Suppose $X$ contains $E C_{3}$-ci. As in the proof of Theorem 3.1, we conclude that, for some $n>N, X_{n}$ contains $E C_{1} C_{3}$-ci. That is, there exists a subspace $F \hookrightarrow X_{n}$ and a complete contraction $u: F \rightarrow E$ with $\left\|u^{-1}\right\|_{\mathrm{cb}} \leq C_{1} C_{3}$. Since $E$ is $C$-injective, there exists a map $\widetilde{u}: X_{n} \rightarrow E$ such that $\left.\widetilde{u}\right|_{F}=u$ and $\|\widetilde{u}\|_{\mathrm{cb}} \leq C$.

As $\operatorname{ex}\left(E^{*}\right)>C_{2}$, there exists an operator $v: E \rightarrow \mathbf{B}$ such that $\|v\|_{\mathrm{cb}}>C_{2}$ and $\left\|v \otimes I_{\mathbf{M}_{n}}\right\|<1$ (to see this, apply Theorem 18 of [15] to $E^{*}$, and dualize). Note that

$$
\|v \widetilde{u}\|_{\mathrm{cb}} \geq\|v u\|_{\mathrm{cb}} \geq \frac{\|v\|_{\mathrm{cb}}}{\left\|u^{-1}\right\|_{\mathrm{cb}}} \geq \frac{C_{2}}{C_{1} C_{3}} .
$$

On the other hand, by definition of $X_{n}$,

$$
\|v \widetilde{u}\|_{\mathrm{cb}}=\left\|v \widetilde{u} \otimes I_{\mathbf{M}_{n}}\right\| \leq\left\|v \otimes I_{\mathbf{M}_{n}}\right\|\|\widetilde{u}\|_{\mathrm{cb}}<C .
$$

This contradicts our assumption that $C_{2} /\left(C_{1} C_{3}\right)>C$.

Corollary 5.6. If $\ell_{\infty}^{n}$ is $C$-BDR, then $C \geq n /(2 \sqrt{n-1})$.

Proof. We know that $E=\ell_{\infty}^{n}$ is 1-injective and 1-exact. Moreover (see Theorem 21.5 of $[16]), \operatorname{ex}\left(E^{*}\right) \geq n /(2 \sqrt{n-1})$. We complete the proof by applying Theorem 5.5.

6. $C^{*}$-algebras and bidual representability. Passing from operator spaces to $C^{*}$-algebras, we obtain:

Proposition 6.1. Suppose $X$ is a $C^{*}$-algebra, and $n \in \mathbb{N}$. Then the following are equivalent:

(1) $X^{* *}$ contains $\mathbf{M}_{n}$ completely isometrically.

(2) $X^{* *}$ contains $\mathbf{M}_{n}$ as a sub-C ${ }^{*}$-algebra.

(3) X contains $\mathbf{M}_{n}$ completely isometrically.

(4) $X^{* *}$ contains $\mathbf{M}_{n}$ c-completely isomorphically for some $c<n /(n-1)$. 
(5) There exists an irreducible representation of $X$ into $B(H)$ with $\operatorname{dim} H \geq n$.

REMARK 6.2. J. Roydor [18] proved a related result: if a $C^{*}$-algebra $X$ embeds into $C\left(\Omega, \mathbf{M}_{n}\right)$ (for some $\Omega$ ) completely isometrically, then $X$ is a $C^{*}$-subalgebra of $C\left(\Omega^{\prime}, \mathbf{M}_{n}\right)$ for some set $\Omega^{\prime}$.

Below, we denote by $\left(E_{i j}\right)_{i, j=1}^{n}$ the canonical matrix units in $\mathbf{M}_{n}$.

Lemma 6.3. Suppose $n>m$, and $T: \mathbf{M}_{n} \rightarrow \mathbf{M}_{m}$ is a complete contraction. Then

$$
\left\|T \otimes I_{\mathbf{M}_{n}}\left(\sum_{i=1}^{n} E_{i j} \otimes E_{i j}\right)\right\|_{\mathbf{M}_{m} \otimes \mathbf{M}_{n}} \leq m .
$$

Corollary 6.4. If $\mathcal{I}$ is a set, $m<n$, and $E$ is an $n^{2}$-dimensional subspace of $\ell_{\infty}\left(\mathcal{I}, \mathbf{M}_{m}\right)$, then $d_{\mathrm{cb}}\left(\mathbf{M}_{n}, E\right) \geq n / m$.

Proof. Consider a complete contraction $T: \mathbf{M}_{n} \rightarrow E$. By Lemma 6.3, $\left\|T \otimes I_{\mathbf{M}_{n}}\left(\sum_{i=1}^{n} E_{i j} \otimes E_{i j}\right)\right\| \leq m$. However, $\left\|\sum_{i, j=1}^{n} E_{i j} \otimes E_{i j}\right\|_{\mathbf{M}_{n} \otimes \mathbf{M}_{n}}=n$.

Proof of Lemma 6.3. Consider a complete contraction $T: \mathbf{M}_{n} \rightarrow \mathbf{M}_{m}$. By Stinespring's representation theorem, there exists a Hilbert space $\widetilde{H}$, a unital representation $\pi: \mathbf{M}_{n} \rightarrow B(\widetilde{H})$, and contractions $U \in B\left(\widetilde{H}, \ell_{2}^{m}\right)$, $V \in B\left(\ell_{2}^{m}, \widetilde{H}\right)$ such that $T a=U \pi(a) V$ for any $a \in \mathbf{M}_{n}$. Note that, for each $i$, $p_{i}=\pi\left(E_{i i}\right)$ is a projection. Denote its range by $H_{i}$. Then $\pi\left(E_{i j}\right)$ is a partial isometry, with initial and terminal projections $H_{i}$ and $H_{j}$, respectively. Let $H=H_{1}$ and $u_{i}=\pi\left(E_{i 1}\right): H \rightarrow H_{i}$ (once again, $\left.1 \leq i \leq n\right)$. Then $\ell_{2}^{n}(H)$ can be identified with $\widetilde{H}$ via $\left(\xi_{i}\right)_{i=1}^{n} \mapsto \sum_{i=1}^{n} u_{i} \xi_{i}$.

For $1 \leq i \leq n$, let $U_{i}=U u_{i} \in B\left(H, \ell_{2}^{m}\right)$ and $V_{i}=u_{i} p_{i} V \in B\left(\ell_{2}^{m}, H\right)$. Then we can identify $U$ with $\sum_{i=1}^{n} E_{1 i} \otimes U_{i}$ (viewed as an operator from $\ell_{2}^{n}(H)$ to $\left.\ell_{2}^{m}\right)$. Similarly, we identify $V$ with $\sum_{i=1}^{n} E_{i 1} \otimes V_{i} \in B\left(\ell_{2}^{m}, \ell_{2}^{n}(H)\right)$. Then

$$
\begin{gathered}
\|V\|=\left\|\sum_{i=1}^{n} E_{i 1} \otimes V_{i}\right\|=\left\|\sum_{i=1}^{n} V_{i}^{*} V_{i}\right\|^{1 / 2} \leq 1, \\
\|U\|=\left\|\sum_{i=1}^{n} E_{1 i} \otimes U_{i}\right\|=\left\|\sum_{i=1}^{n} U_{i} U_{i}^{*}\right\|^{1 / 2} \leq 1 .
\end{gathered}
$$

Moreover, $T E_{i j}=U_{i} V_{j}$ for any $i$ and $j$. Therefore,

$$
\begin{aligned}
\left\|T \otimes I_{\mathbf{M}_{n}}\left(\sum_{i, j=1}^{n} E_{i j} \otimes E_{i j}\right)\right\|_{\mathbf{M}_{m} \otimes \mathbf{M}_{n}} & =\left\|\sum_{i, j=1}^{n} U_{i} V_{j} \otimes E_{i j}\right\| \\
& =\left\|\left(\sum_{i} U_{i} \otimes E_{i 1}\right)\left(\sum_{j} V_{j} \otimes E_{1 j}\right)\right\| .
\end{aligned}
$$


However,

$$
\begin{aligned}
\left\|\sum_{i} U_{i} \otimes E_{i 1}\right\| & =\left\|\sum_{i=1}^{n} U_{i}^{*} U_{i}\right\|^{1 / 2} \leq\left(\operatorname{Tr}\left(\sum_{i=1}^{n} U_{i}^{*} U_{i}\right)\right)^{1 / 2} \\
& =\left(\operatorname{Tr}\left(\sum_{i=1}^{n} U_{i} U_{i}^{*}\right)\right)^{1 / 2} \leq \sqrt{m}\left\|\sum_{i=1}^{n} U_{i} U_{i}^{*}\right\| \leq \sqrt{m}
\end{aligned}
$$

and similarly, $\left\|\sum_{j} V_{j} \otimes E_{1 j}\right\| \leq \sqrt{m}$. Thus,

$$
\left\|T \otimes I_{\mathbf{M}_{n}}\left(\sum_{i, j=1}^{n} E_{i j} \otimes E_{i j}\right)\right\| \leq\left\|\sum_{i} U_{i} \otimes E_{i 1}\right\| \cdot\left\|\sum_{j} V_{j} \otimes E_{1 j}\right\| \leq m .
$$

REMARK 6.5. In a similar fashion, one can show that

$$
\left\|\sum_{i=1}^{n} E_{i 1} \otimes E_{i 1}\right\|_{\mathbf{C}_{n} \otimes \mathbf{C}_{n}}=\sqrt{n}
$$

and

$$
\left\|T \otimes I_{\mathbf{C}_{n}}\left(\sum_{i=1}^{n} E_{i 1} \otimes E_{i 1}\right)\right\|_{\mathbf{M}_{m} \otimes \mathbf{C}_{n}} \leq \sqrt{m}
$$

whenever $T: \mathbf{C}_{n} \rightarrow \mathbf{M}_{m}$ is a complete contraction. From this, one concludes that $d_{\mathrm{cb}}\left(\mathbf{C}_{n}, E\right) \geq \sqrt{n / m}$ for every $n$-dimensional subspace $E$ of $\ell_{\infty}\left(\mathcal{I}, \mathbf{M}_{m}\right)$ (provided $n>m$ ).

Lemma 6.6. Suppose $\pi: Y \rightarrow B(H)$ is an irreducible representation of a $C^{*}$-algebra $Y$ on a Hilbert space $H$ of dimension at least $n$. Then $Y$ contains $\mathbf{M}_{n}$ completely isometrically as an operator system.

Proof. If $p$ is a projection of rank $n$ on $H$, then, by the transitivity of irreducible representations (Theorem II.4.18 of [20]), $\pi(Y) p=B(H) p$. Denote by $A$ the set of all $y \in Y$ satisfying $\pi(y) p=p \pi(y)(=p \pi(y) p)$. Clearly, $A$ is a $C^{*}$-subalgebra of $Y$, and $\pi(A)$ can be identified with $B(p(H)) \sim \mathbf{M}_{n}$. Moreover, $A$ has a separable subalgebra (call it $A_{1}$ ) such that $\pi\left(A_{1}\right)$ can again be identified with $\mathbf{M}_{n}$. In other words, $\mathbf{M}_{n}=A_{1} / J$, where $J=\operatorname{ker} \pi \cap A_{1}$ is a closed two-sided ideal. By Theorem 3.10 of [3], $\mathbf{M}_{n}$ lifts to $A_{1}$ completely positively. Thus, $A_{1}$ contains $\mathbf{M}_{n}$ completely isometrically.

REMARK 6.7. A similar lifting technique was used in [7]. Earlier, lifting methods were used in $[19,21]$ to prove that a $C^{*}$-algebra which is not $(n-1)$ subhomogeneous contains a completely positive copy of $\mathbf{M}_{n}$.

Proof of Proposition 6.1. The implications $(2) \Rightarrow(1) \Rightarrow(4)$ and $(3) \Rightarrow(1)$ are clear.

$(1) \Rightarrow(2)$ : By Section 6.4 of [10], we can decompose the von Neumann algebra $X^{* *}$ into a direct sum of components of type $\mathrm{I}_{k}(k \in \mathbb{N} \cup\{\infty\})$, II, and III. By Lemma 6.4, at least one of the summands of type $\mathrm{I}_{k}(k \geq n)$, 
II, and III is non-trivial. All such summands contain $\mathbf{M}_{n}$ as a subalgebra (see e.g. comparison theorem for projections in a von Neumann algebra, Theorem 6.2 .7 of [10]).

$(1) \Rightarrow(5)$ : Suppose, for the sake of contradiction, that (5) fails to hold. Viewing $X^{* *}$ as the enveloping algebra of $X$, we embed it into $\ell_{\infty}\left(\mathcal{I}, \mathbf{M}_{n-1}\right)$ for some set $\mathcal{I}$. Therefore, by Lemma $6.4, X^{* *}$ cannot contain $\mathbf{M}_{n} c$-completely isomorphically with $c<n /(n-1)$.

$(4) \Rightarrow(1)$ and $(5) \Rightarrow(3)$ : If there are no irreducible representations of $X^{* *}$ (or $X$ ) on Hilbert spaces with dimension $\geq n$, then $X^{* *}$ (respectively, $X$ ) embeds into $\ell_{\infty}\left(\mathcal{I}, \mathbf{M}_{n-1}\right)$, hence, by Lemma $6.4, X^{* *}$ cannot contain $\mathbf{M}_{n}$ $c$-completely isomorphically when $c<n /(n-1)$. Otherwise, $X^{* *}$ (or $X$ ) contains $\mathbf{M}_{n}$ completely isometrically, by Lemma 6.6 .

Remark 6.8. By Lemma 6.6, items (1) and (4) of Proposition 6.1 guarantee that $X$ (or $X^{* *}$ ) contains $\mathbf{M}_{n}$ as an operator system. However, $X$ need not contain $\mathbf{M}_{n}$ as a subalgebra. Indeed, let $X$ be the left regular algebra of a free group on two generators. By [4], $X$ has no non-trivial projections, hence it cannot contain $\mathbf{M}_{n}$ as a subalgebra.

Acknowledgments. We are grateful to $\mathrm{H}$. Rosenthal for suggesting the topic of this paper, and to M. Junge and R. Smith for useful conversations. We would like to thank the organizers of the workshop in Linear Analysis and Probability in College Station, TX, where part of this work was carried out. We thank the referee for bringing [18] to our attention.

\section{References}

[1] A. Arias and H. Rosenthal, $M$-complete approximate identities in operator spaces, Studia Math. 141 (200), 143-200.

[2] D. Blecher and C. le Merdy, Operator Algebras and Their Modules-an Operator Space Approach, Oxford Univ. Press, New York, 2004.

[3] M.-D. Choi and E. Effros, The completely positive lifting problem for $C^{*}$-algebras, Ann. of Math. 104 (1976), 585-609.

[4] K. Davidson, $C^{*}$-algebras by Example, Amer. Math. Soc., Providence, RI, 1996.

[5] E. Effros and Z.-J. Ruan, Mapping spaces and liftings for operator spaces, Proc. London Math. Soc. 69 (1994), 171-197.

[6] - - -, Operator Spaces, Oxford Univ. Press, Oxford, 2000.

[7] F. Hansen, G. Ji, and J. Tomiyama, Gaps between classes of matrix monotone functions, Bull. London Math. Soc. 36 (2004), 53-58.

[8] P. Harmand, D. Werner, and W. Werner, M-ideals in Banach Spaces and Banach Algebras, Lecture Notes in Math. 1547, Springer, Berlin, 1993.

[9] W. Johnson, H. Rosenthal, and M. Zippin, On bases, finite-dimensional decompositions and weaker structures in Banach spaces, Israel J. Math. 9 (1971), 488-506.

[10] R. Kadison and J. Ringrose, Fundamentals of the Theory of Operator Algebras I, Amer. Math. Soc., Providence, RI, 1997. 
[11] F. Lehner, $M_{n}$ espaces, sommes d'unitaires et analyse harmonique sur le groupe libre, PhD thesis, Univ. Paris VI, 1997.

[12] J. Lindenstrauss and H. Rosenthal, The $\mathcal{L}_{p}$ spaces, Israel J. Math. 7 (1969), 325-349.

[13] T. Oikhberg and E. Ricard, Operator spaces with few completely bounded maps, Math. Ann. 328 (2004), 229-259.

[14] V. Paulsen, Completely Bounded Maps and Operator Algebras, Cambridge Univ. Press, 2002.

[15] G. Pisier, Exact operator spaces, Astérisque 232 (1995), 159-186.

[16] - An Introduction to the Theory of Operator Spaces, Cambridge Univ. Press, 2003.

[17] G. Pisier and D. Shlyakhtenko, Grothendieck's theorem for operator spaces, Invent. Math. 150 (2002), 185-217.

[18] J. Roydor, Subalgebras of $C\left(\Omega, M_{n}\right)$ and their modules, Illinois J. Math. 49 (2005), 1019-1038.

[19] R. Smith, Completely bounded maps between $C^{*}$-algebras, J. London Math. Soc. 27 (1983), 157-166.

[20] M. Takesaki, Theory of Operator Algebras I, Springer, 2002.

[21] J. Tomiyama, On the difference of n-positivity and complete positivity in $C^{*}$-algebras, J. Funct. Anal. 49 (1982), 1-9.

Department of Mathematics

University of Denver

Denver, CO 80208, U.S.A.

E-mail: arias@math.du.edu
Department of Mathematics The University of California at Irvine Irvine, CA 92697-3875, U.S.A. E-mail: toikhber@math.uci.edu

Received June 27, 2006

Revised version May 29, 2007 